Musées, Patrimoine et Culture scientifiques et techniques

$141 \mid 2012$

mai - juin 2012

\title{
Expérience de visite, identités et self-aspects
}

Visitor experiences, identities and self-aspects

John-H. Falk

URL : http://journals.openedition.org/ocim/1061

DOI : 10.4000/ocim. 1061

ISSN : 2108-646X

Éditeur

OCIM

Édition imprimée

Date de publication : 1 mai 2012

Pagination : 5-14

ISSN : 0994-1908

Référence électronique

John-H. Falk, «Expérience de visite, identités et self-aspects », La Lettre de l'OCIM [En ligne], 141 | 2012, mis en ligne le 01 mai 2014, consulté le 01 mai 2019. URL : http://journals.openedition.org/ocim/1061 ; DOI : 10.4000/ocim.1061 


\section{Expérience de visite, identités et self-aspects}

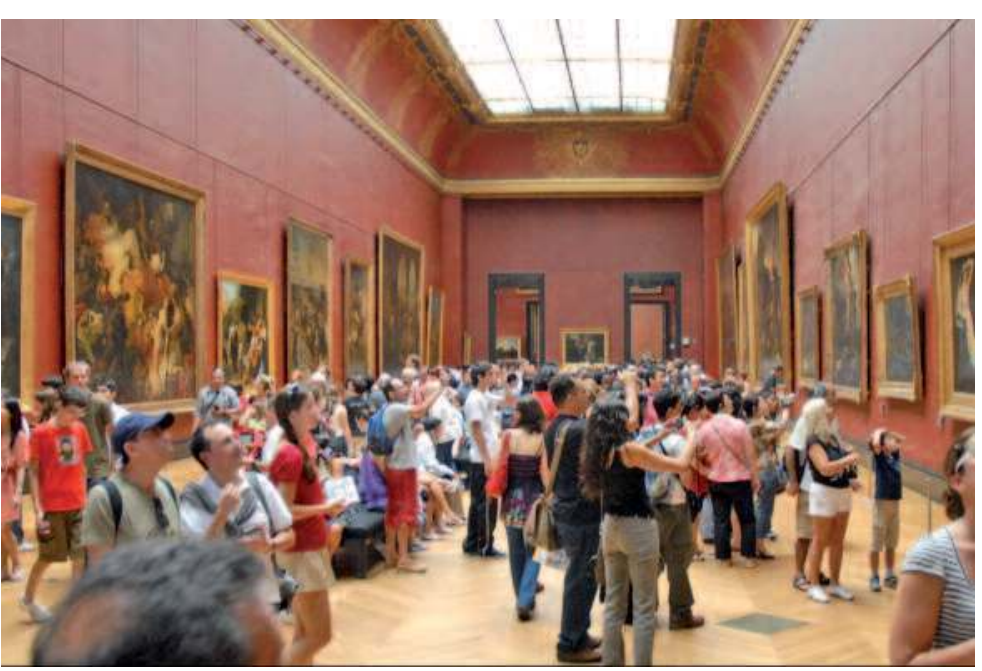

Visites d'été au musée du Louvre à Paris (c) Flickr/Dalbera

\footnotetext{
* John-H. Falk est directeur du centre de recherche Lifelong STEM Learning et professeur de Free-Choice Learning, Oregon State University, États-Unis
}

\begin{abstract}
John-H. Falk *
À partir d'une critique des concepts et des méthodes habituellement utilisés pour conduire des études de publics, l'auteur propose une nouvelle approche qui, au-delà des découpages en catégories socioprofessionnelles hérités de la tradition néomarxiste, prend davantage en compte les différentes facettes de l'identité du visiteur : la conscience réflexive de la diversité des rôles que celui-ci assume selon la situation ou le contexte fait qu'il ne visite pas toujours de la même façon un musée ou une exposition.

Cet article est le premier texte publié en langue française de John-H. Falk, spécialiste de la recherche sur les musées de ce qu'il est convenu d'appeler "l'expérience de visite ». La traduction et l'adaptation à la langue française ont été réalisées par Jason Luckeroff et Daniel Jacobi.
\end{abstract}

Pourquoi la recherche ne parvient-elle pas à prédire l'expérience de visite?

Depuis plusieurs décennies, les chercheurs s'efforcent de décrire et de comprendre ce qu'on appelle l'expérience de visite. Pourtant, deux problèmes majeurs limitent la validité et la fiabilité d'une grande partie de leurs travaux. Le premier est d'ordre spatial : la quasi-totalité des recherches sur la fréquentation des musées ont été menées à l'intérieur du musée. $\mathrm{Si}$ on veut comprendre quelque chose à propos des visiteurs de musée, il paraît logique de les interroger pendant qu'ils font la visite du musée. Et quel est 
l'endroit le plus propice pour rencontrer les visiteurs ? À l'intérieur du musée, bien entendu.

Le second est en apparence d'ordre temporel mais en réalité plus conceptuel. Interroger le public seulement dans le musée sous-entend implicitement que seule la visite elle-même détermine la nature de l'expérience. En réalité, il n'en est rien. Une partie seulement de l'expérience de visite se passe entre les quatre murs du musée. La décison de venir au musée, et pour quel motif, est construite hors du musée et avant la visite. Ce que le visiteur apporte avec lui et en particulier son expérience antérieure, ses connaissances et ses propres intérêts influence profondément ce qu'il fait ou ce qu'il ressent pendant la visite. En outre, la plupart des visiteurs arrivent en compagnie d'un petit groupe d'autres visteurs. Ce groupe social influence considérablement le cours et le contenu de l'expérience de visite. Or, la recherche dans ce domaine a montré que la plupart des interactions sociales qui surviennent dans un musée sont en fait directement liées à des conversations et des relations que les visiteurs ont engagé avant même d'entrer dans le musée. En d'autres mots, il n'est pas possible de comprendre pleinement ce que quelqu'un fait dans le musée et pourquoi il le fait à moins de connaître quelque chose de la vie de cette personne avant son arrivée dans le musée.

Les significations que les gens attribuent à leur expérience de visite d'un musée s'étendent également audelà des limites temporelles et spatiales du musée. Ce n'est que récemment que nous avons découvert combien un temps de latence est nécessaire pour que des souvenirs se fixent dans la mémoire (McGaugh, 2003). Cela peut prendre des jours, parfois même des semaines pour qu'un souvenir se pérennise, et pendant ce temps-là d'autres expériences et d'autres événements interviennent qui peuvent modifier l'empreinte mnésique. De même que les conversations commencent avant la visite, elles peuvent également se poursuivre après que les visiteurs aient quitté le musée. Paradoxalement, ce qui se passe après qu'une personne ait quitté le musée peut être aussi déterminant quant à la nature et la permanence de la mémoire de visite que ce qui s'est réellement passé au sein du musée.

Interroger un visiteur pendant la visite ou immédiatement à la sortie du musée ne saurait refléter véritablement et précisément la nature complète de son expérience de visite dans la mesure où cela revient à ignorer tout le processus d'élaboration mentale qui s'est produit à la suite d'une visite. Par conséquent, les visiteurs sont littéralement incapables de décrire avec soin ce qu'ils ont ou n'ont pas réellement appris. Comprendre l'expérience de visite de musée

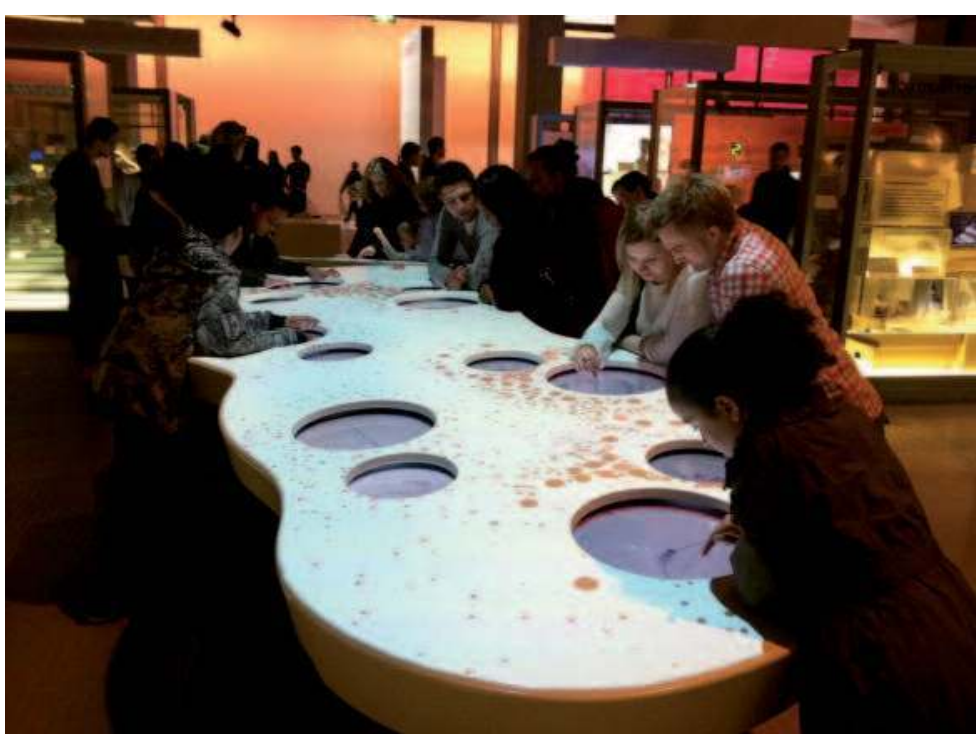

Les « Explorateurs », au Science Museum de Londres (c) Flickr/Coolinsights

réclame l'allongement du délai d'enquête de manière à pouvoir y inclure les aspects de la vie du visiteur relevant de l'avant-visite et l'après-visite du musée.

\section{Les limites des enquêtes de public}

Deux autres traditions empiriques que nous évoquerons successivement, s'opposent à la production de connaissances nouvelles sur l'expérience de visite. La première a trait à la prépondérance des contenus sur l'expérience de visite. Le goût et la prédilection pour les beaux-arts, l'archéologie, l'histoire ou les sciences et techniques seraient à la fois ce qui motive la visite et ce qui facilite ce qu'on apprend et retient à l'issue de celle-ci. De multiples recherches, y compris celles de notre équipe, vont dans ce sens. Pourtant, la population qui aime, apprécie et goûte tel ou tel contenu va très au-delà du nombre de ceux qui se rendent dans un musée ou une exposition spécialisée. Ce qui signifie que, s’il est nécessaire d'avoir un intérêt pour le contenu d'une exposition, cela n'est pas suffisant pour savoir qui va fréquenter, ou ne pas fréquenter un musée donné, et encore moins pour prédire qui va le fréquenter tel ou tel jour.

Certes, le contenu d'une exposition est très important, mais il ne saurait seul rendre compte de la nature de l'expérience de visite. Selon l’une de nos études, effectuée il y a déjà longtemps, un visiteur consacrait, au cours d'une visite, à peu près $60 \%$ de son attention à regarder le contenu proprement dit de l'exposition, avec une concentration intense pendant le premier quart d'heure de la visite (Falk et al., 1985), 
attention qui s'effiloche ensuite. Cela signifie qu'environ $40 \%$ de l'attention du visiteur se porte ailleurs, principalement sur des conversations avec les personnes qui l'accompagnent ou sur des observations portant sur le lieu, d'autres visiteurs ou des éléments périphériques. Certes, le contenu guide fortement l'expérience de visite, mais en aucun cas il ne le régule en totalité. Et bien entendu, le contenu, sur lequel le visiteur choisit de se concentrer, peut ou non ressembler au contenu que les professionnels ont produit et à l'expérience qu'ils espéraient susciter. Ce qui nous amène évidemment à la question suivante : en définitive, quel est le contenu que le visiteur retient à long terme d'une exposition ? L'une de nos recherches a révélé que, pour certains visiteurs (mais jamais pour tous les visiteurs), ce qui était retenu était lié à la qualité de l'exposition (Falk \& Storksdieck, 2005). Dans certains cas, les visiteurs qui se sont rendus dans des expositions de bonne qualité (celles qui communiquent le contenu prévu de façon claire et attrayante) apprennent davantage. Dans d'autres cas, l'apprentissage semble être totalement indépendant du niveau de qualité des expositions. Ce qui démontre que l'expérience de visite est influencée par la nature du musée et la qualité de ses expositions, mais pas exclusivement.

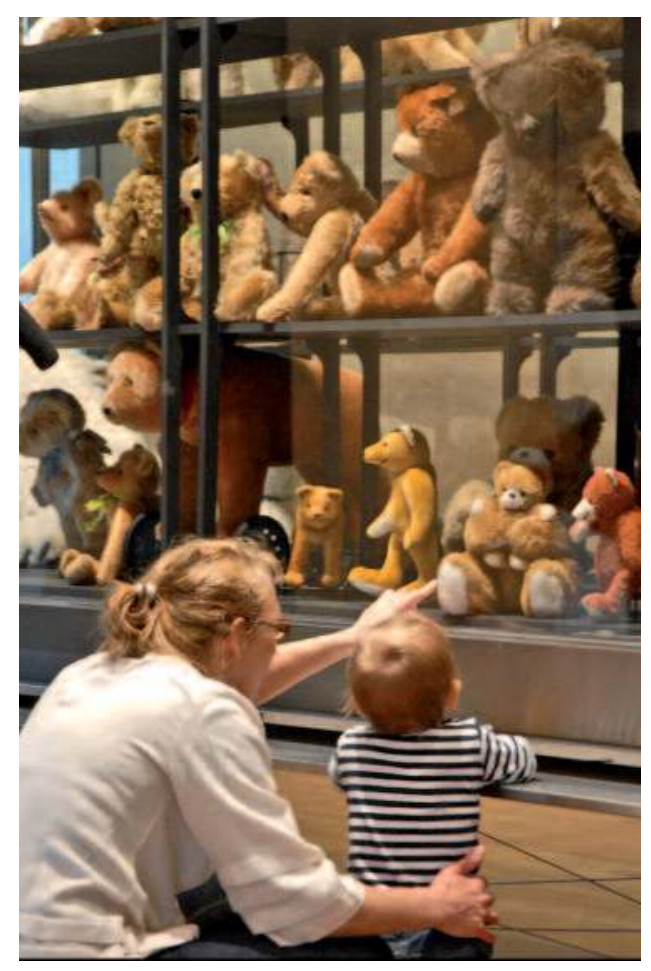

Les « Facilitateurs », au musée des Arts décoratifs à Paris ๑) Flickr/Dalbera
La seconde tradition empirique est héritée de la tradition sociologique et du découpage en classes. Au cours des dernières décennies, des milliers d'études sur les visiteurs ont été menées afin de mieux comprendre qui fréquente les musées. Bien que seule une infime fraction de ces études ait été publiée, quasiment tous les musées, de la maison-musée minuscule, à l'aire naturelle gérée par des bénévoles, en passant par le musée d'Histoire naturelle, le zoo, l'aquarium et le centre des sciences, ont tenté de décrire qui sont leurs visiteurs. La très grande majorité de ces enquêtes a utilisé, d'une part, des variables socio-démographiques classiques telles que l'âge, la scolarité, le sexe, le groupe d'appartenance... autrement dit des paramètres stables, qui ne varient pas d'un jour à l'autre et qui auraient une valeur explicative du comportement. D'autre part, on a utilisé d'autres variables comme la fréquence des visites (fréquentes, occasionnelles, non-visiteur...) et la sociabilité (famille ou parents, amis, groupe scolaire...). Par conséquent, nous savons beaucoup de choses sur certaines caractéristiques du visiteur de musée, celles que les agences gouvernementales et les chercheurs en sciences sociales ont traditionnellement utilisées pour décrire et catégoriser les publics ${ }^{(1)}$.

L'un des résultats prévisibles de la segmentation des groupes en différentes catégories socio-démographiques mesurables est que des tendances se dessinent; que ces modèles soient significatifs ou non est une autre question. Il n'est donc peut-être pas surprenant qu'un certain nombre de variables démographiques aient été corrélées positivement avec la visite du musée comme le niveau de diplôme, le revenu, la profession, le groupe d'appartenance et l'âge. Un constat assez fréquent montre que les amateurs de musée sont plus instruits, plus fortunés, et ont des emplois mieux rémunérés que la moyenne de leurs concitoyens. Ceci est vrai des visiteurs de musées d'art, d'histoire et de science ainsi que des visiteurs de zoos, jardins botaniques et parcs nationaux. Comme cela a été démontré il y a déjà longtemps (en particulier par Bourdieu et Darbel, 1991/1969), l'appartenance à une classe sociale semble être déterminante. Aux États-Unis, l'autre variable démographique qui a été intensivement utilisée est la couleur de peau ou l'appartenance à un groupe d'immigration plus récente (afros, latinos, asiatiques...). Dans une enquête longitudinale sur la fréquentation des musées par les Afro-Américains, nous avons montré que leur appartenance à cette catégorie de la population ne permet pas de connaître les raisons pour lesquelles les Noirs américains fréquentaient ou non des musées 
(Falk, 1993). D’autres recherches, à Los Angeles, ont confirmé que l'origine, pas plus que l'âge et le niveau d'éducation n'étaient des bons prédicteurs de la fréquentation d'un musée en particulier (Falk et Needham, 2011).

Les paramètres sociologiques quantifiables ne donnent que l'impression d'expliquer. Nous savons que les visiteurs de musées sont plus scolarisés, plus âgés, plus blancs, plus aisés et plus féminins que le public en général, mais qu’est-ce que cela signifie réellement? Même si ces statistiques sont en moyenne exactes, les visiteurs de musée, eux, ne sont pas des moyennes : ce sont des individus (2). Savoir que quelqu'un est plus scolarisé, plus âgé, plus blanc, plus riche et plus féminin que le public dans son ensemble fournit des renseignements insuffisants pour prédire si oui ou non il va fréquenter un musée. De même, savoir que quelqu'un est moins scolarisé, plus jeune, plus brun, plus pauvre et plus masculin que le public de musée en général ne fournit que des renseignements insuffisants pour prédire qu'il ne fréquentera pas de musée.

En fait, la principale conclusion de nos travaux, après avoir étudié des milliers de visiteurs pendant plus de trois décennies, est que la fréquentation muséale est beaucoup trop complexe pour être comprise uniquement sur la base de variables concrètes, faciles à mesurer, telles que les données socio-démographiques ou par ailleurs des qualités tangibles comme « le type de musée » ou « le genre muséographique » (par exemple, pratique, didactique, interactif...). L'expérience de visite ne peut pas être réduite et expliquée par des variables immuables. L'expérience de visite de musée est bien trop éphémère et dynamique : c'est une relation qui se construit de façon unique, chaque fois qu'une personne entre dans un musée.

\section{Un nouveau modèle de l'expérience de visite}

Comment mieux approcher l'expérience de visite ? Une centaine d'entretiens en profondeur administrés à des visiteurs, des semaines, des mois et des années après leur visite du musée, ont servi de base à cette recherche. Ce qui ressort nettement de ces entretiens est le côté profondément personnel des visites de musées, et combien pour chaque individu celle-ci est liée à son identité. Dans son récit, il se sert des mêmes mots pour décrire ses souvenirs d'avant et d'après la visite. Ce qu'il dit des motivations, qui l'ont conduit à se rendre au musée, correspond exactement à ce qu'il a ressenti pendant la viste et

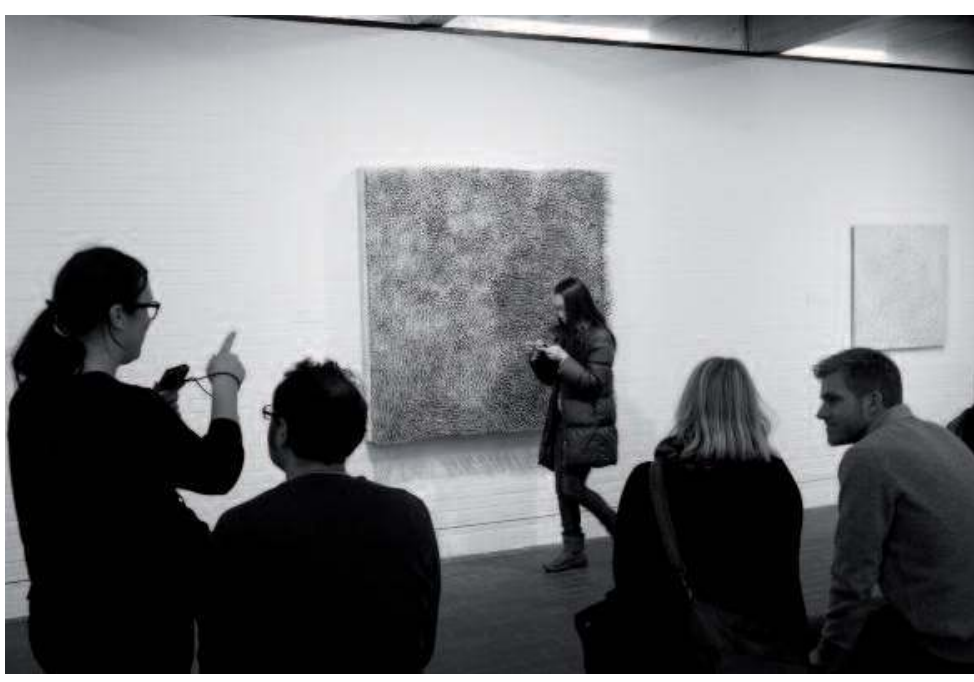

Les «Professionnels/et Amateurs avertis », au museum of Modern Art du Danemark (c) Flickr/Sigfrid Lundberg

dont il se souvient en mémoire à long terme après la visite. Les leçons tirées de ces entretiens m'ont amené à totalement repenser l'expérience de visite. La quasitotalité des visites de musées est configurée autour de l'identité personnelle du visiteur et particulièrement de sa construction ou de son renforcement ${ }^{(3)}$.

Un grand nombre de chercheurs ont cherché à décrire les différentes catégories de motivations de ceux qui visitent des musées. Plus récemment, les enquêteurs ont commencé à rechercher les relations entre leurs motivations initiales et le sens qu'ils attribuent à leur visite à leur sortie. Comme l'ont postulé Doering et Pekarik (1996), les visiteurs viennent dans un musée avec "un récit d'entrée » qui est susceptible de se renforcer, en guidant directement à la fois le comportement, l'apprentissage et les perceptions de satisfaction. Nos entretiens approfondis vont dans le même sens. Mais de plus, j'ai repéré un schéma robuste et récurrent dans ces récits d'entrée. Chacun de ces récits, parmi la centaine que j'ai recueillie, était unique ; mais en prenant un peu de recul, il était possible d'y voir une régularité : tous décrivent les motivations d'une personne singulière. Et toutes ces motivations pourraient être mieux comprises comme visant à satisfaire un ou plusieurs buts et besoins liés à l'identité personnelle.

Depuis plus de cent ans, les éléments de construction du soi et de l'identité ont été utilisés par un large éventail de chercheurs en sciences sociales provenant de disciplines variées. Il n'est peut-être pas surprenant, de ce fait, qu'il n'existe pas de définition consensuelle du soi ou de l'identité, bien qu'il y ait 
un certain nombre de recensions utiles décrivant ces différentes perspectives (Falk, 2009). Soulignant la complexité du sujet, Bruner et Kalmar (1998, p. 326) déclarent : "Le soi est à la fois extérieur et intérieur, public et privé, inné et acquis, le produit de l'évolution et le fruit d'un récit formé par la culture». Il a été caractérisé comme le produit d'un dialogue sans fin et d'une comparaison avec les «autres », à la fois vivants et non-vivants (Bakhtine, 1981). Peut-être plus à propos, Simon $(2004$, p. 3) stipule : "même si l'identité se révèle être une "fiction analytique", elle se révélera comme étant une fiction analytique très utile dans la recherche d'une meilleure compréhension des expériences et des comportements humains. (...), la notion d'identité aura comme fonction d'être un puissant outil conceptuel».

Je souscris à l'idée que l'identité est au confluent de forces sociales internes et externes - agents culturels et individuels (Falk, 2009). Je crois que l'identité est toujours influencée, plus ou moins, par des perceptions innées et acquises. Je souscris aussi à l'idée que la construction du soi est un processus sans fin, sans limite temporelle claire. Dans cette perspective, l'identité apparaît comme malléable, sans cesse construite, et comme une qualité qui se situe toujours dans les réalités du monde matériel et socioculturel. Dans le monde physique et social immédiat, dans lequel navigue un individu, mais aussi dans le monde social et physique plus large d'un individu incluant sa famille passée (et future), sa culture et son histoire personnelle.

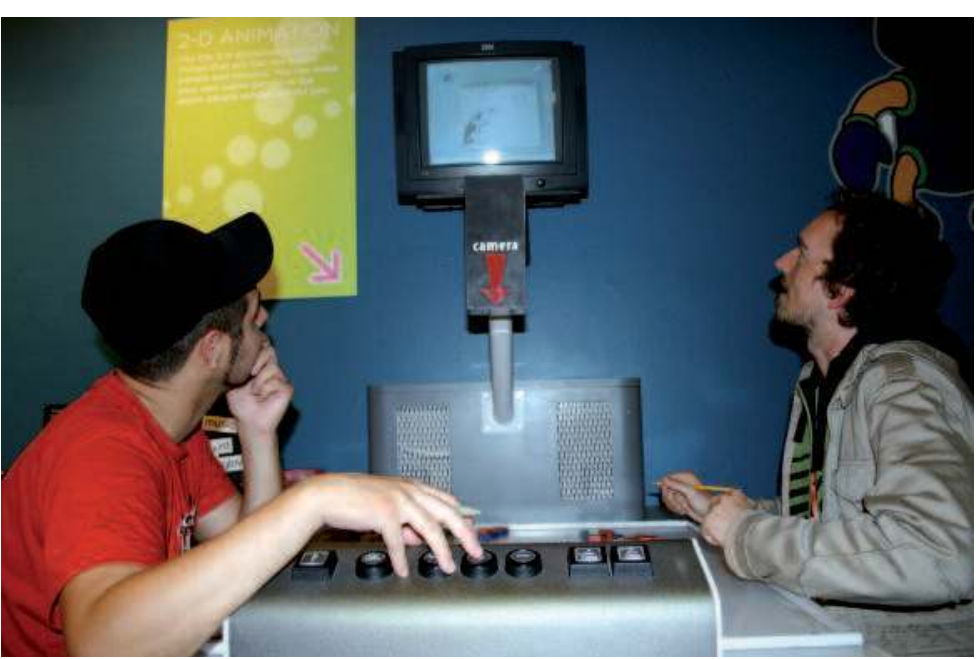

Les «Curieux d'expériences», à I'Exploratorium de San Franscico (C) Flickr/Mskogly
Une compréhension clé de l'identité est que chacun d'entre nous n'a pas une identité unique, mais maintient plutôt de nombreuses identités qui s'expriment collectivement ou individuellement à des moments différents, selon le besoin et les circonstances. Chacun de nous possède et agit sur un ensemble d'identités durables et profondes (ce que j'appelle les Identités avec un grand «I »). Des exemples d'identités avec un grand «I » pourraient être l'idée de son genre, sa nationalité, ses opinions politiques ou sa religion : ce sont ces identités que nous portons en nous toute notre vie et bien qu'elles évoluent incontestablement, elles restent relativement constantes (par exemple, la plupart d'entre nous ne changeons pas notre idée de genre ou de nationalité, même si notre idée de ce que le genre ou la nationalité représente évolue). Ce sont ces types d'identité qui ont été le plus fréquemment étudiés par les sociologues et qui sautent à l'esprit le plus fréquemment quand on pense à l'identité.

Toutefois, je dirais qu'une grande partie de notre vie est consacrée à adopter une série d'autres identités plus localisées qui représentent les réponses aux besoins et aux réalités d'un moment et de circonstances spécifiques (ce que j'appelle les identités avec un petit « $\mathrm{i} »)$. Des exemples d'identités avec un petit « $\mathrm{i} »$ pourraient être l'identité du «bon neveu » que nous adoptons lorsque nous nous souvenons d'envoyer une carte d'anniversaire à notre tante qui vit dans autre une ville ou celle de "l'hôte ou de l'hôtesse » que nous adoptons quand quelqu'un vient nous rendre visite pour la première fois. Si nous étions sur le point d'obtenir le prix Nobel et que quelqu'un nous interviewait, ces sortes d'identités avec un petit « $\mathrm{i} »$ ne seraient pas susceptibles d'arriver en haut de notre liste de caractéristiques que nous offrons comme descripteurs de « qui nous sommes »; mais indéniablement, ces types d'identités jouent un rôle essentiel dans la définition de qui nous sommes et de notre comportement la plupart du temps.

\section{Self et self-aspects (4)}

J'ai observé que pour la plupart des gens, la plupart du temps, aller dans un musée tend à susciter pour la majorité des identités avec un petit « i ». En d'autres termes, les gens vont dans les musées afin de favoriser des besoins liés à leur identité tels que le désir d'être un parent ou un conjoint solidaire, de satisfaire leur sens de la curiosité ou le sentiment qu'il serait bon de sortir de la foire d'empoigne pendant un peu de temps. La nationalité, la religion, le genre ou l'appartenance politique ne semblent pas 


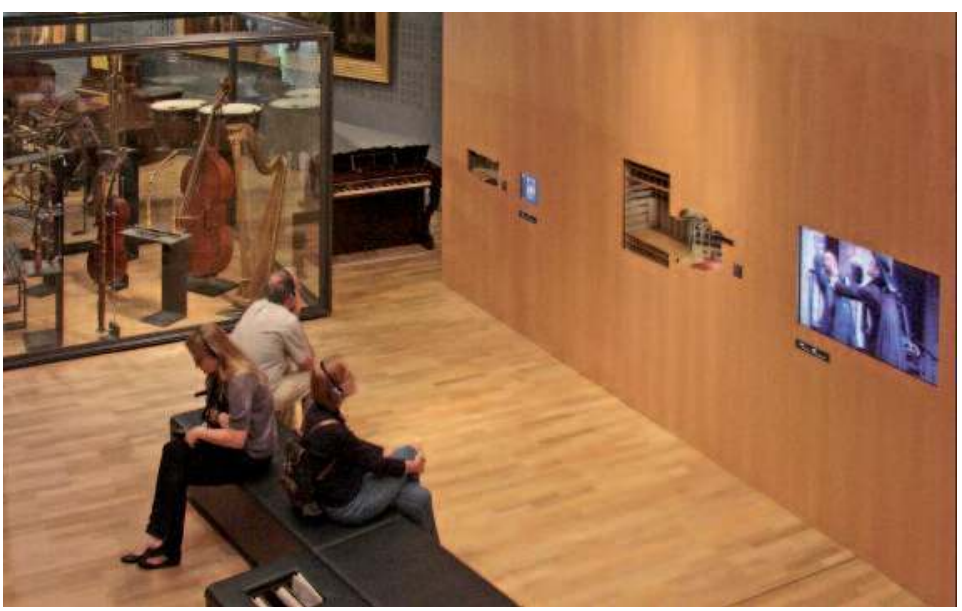

Les « Ressourceurs», dans l'espace consacré à l'opéra à la Cité de la Musique à Paris. () Flickr/Dalbera

être les motifs principaux qui provoquent la plupart des visites de ces personnes dans les musées des beaux-arts, les musées pour enfants, les zoos ou les centres de sciences.

Selon Simon (2004, p. 45), "grâce à l'auto-interprétation, les gens atteignent une compréhension d'euxmêmes ou, en d'autres termes, une identité, qui influence à son tour leur perception et comportement ultérieurs ». Dans le modèle de Simon, la self-interpretation implique un nombre variable de self-aspects, une catégorie cognitive ou un concept qui sert à traiter et organiser l'information et les connaissances sur soi-même. Selon Simon (2004, p. 46), les self-aspects peuvent référer à « des caractéristiques ou traits psychologiques généraux (par exemple, introverti), des caractéristiques physiques (par exemple, cheveux roux), les rôles (par exemple, père), les capacités (par exemple, bilingue), les goûts (par exemple, préférence pour les vins rouges français), les attitudes (par exemple, contre la peine de mort), les comportements (par exemple, je travaille beancoup), et l'appartenance explicite à un groupe ou une catégorie (par exemple, membre du parti communiste) ».

En d'autres termes, dans une situation spécifique, les individus donnent une signification à leurs actions et à leurs rôles en s'attribuant des qualités liées à leur identité. Les travaux de nombreux chercheurs vont dans ce sens. Ils ont constaté que les personnes construisent effectivement des prototypes situationnels, pertinents avec l'identité qu'ils ont adoptée. Elle guide la personne, en lui disant à quoi s'attendre et comment se comporter dans les situations d'un type particulier. J'ai pensé que c'était aussi le cas pour les visiteurs de musées.
Les gens qui visitent les musées ont intégré un modèle de travail de ce qu'implique la visite de musée; ils ont aussi un sens des avantages que la visite leur apportera. Les visiteurs attribuent à leurs expériences de visite un ensemble de self-aspects qui s'articulent autour de la perception qu'ils ont de ce que pourrait leur apporter l'expérience de musées. Les self-aspects des visiteurs seraient donc congruents avec, d'une part, leur compréhension de ce que le musée a à leur offrir, et d'autre part, avec la perception de leurs propres rôles et besoins liés à l'identité. Comme le décrit Erikson (1968), les individus n'ont d'autre choix que de former leur identité en utilisant comme cadre de travail « la gamme existante de solutions de rechange pour la formation de l'identité » (Erikson, 1968, p. 190). Nous avons des preuves qui vont toutes dans ce sens : les visiteurs utilisent leur selfaspect de pré-visite à la fois pour justifier potentiellement pourquoi ils devraient visiter le musée, puis de nouveau rétrospectivement afin de définir en quoi leur visite en valait la peine.

Par exemple, de nombreux visiteurs de musée des beaux-arts se décrivent comme des gens curieux, généralement intéressés par l'art. Ils voient les musées des beaux-arts en tant qu'endroits intéressants pour exercer cette curiosité et cet intérêt. Lorsqu'une personne en particulier est interrogée au sujet des musées des beaux-arts, elle répond : "Les musées des beaux-arts sont des endroits intéressants à visiter, car ils rassemblent des expositions conçues pour cultiver l'intérêt des gens et leur compréhension de l'art». Lorsqu'on lui demande pourquoi elle fréquente ce musée des beaux-arts ce jour-là, elle répond : "Je suis venue voir ce qui est nowveau ici. Cela fait un moment que je n'y suis pas venue et j'espérais voir de l'art vraiment nowveau et intéressant $»$. Plusieurs mois plus tard, quand j’ai réinterrogé cette personne, elle réfléchit à sa visite et dit : "J'ai passé un très bon moment au musée des beaux-arts; je me suis simplement promenée et j'ai vu tout cet art fabuleux; il y avait quelques œevvres vraiment frappantes. J'ai même découvert quelques œuvres que je n'avais jamais vues ou dont je ne savais rien auparavant. C'était vraiment merveilleux».

La compréhension qu'a le visiteur de son expérience de visite est invariablement self-référentielle. Elle lui donne sens et cohérence. Les visiteurs ont tendance à évoquer leur comportement dans le musée et les résultats après leur visite comme conformes à leur identité, c'est-à-dire aux traits personnels, aux attitudes et à leur affiliation à un groupe. Cette visiteuse fait de son expérience de visite un renforcement du point de vue qu'elle a d'elle-même comme étant une personne 


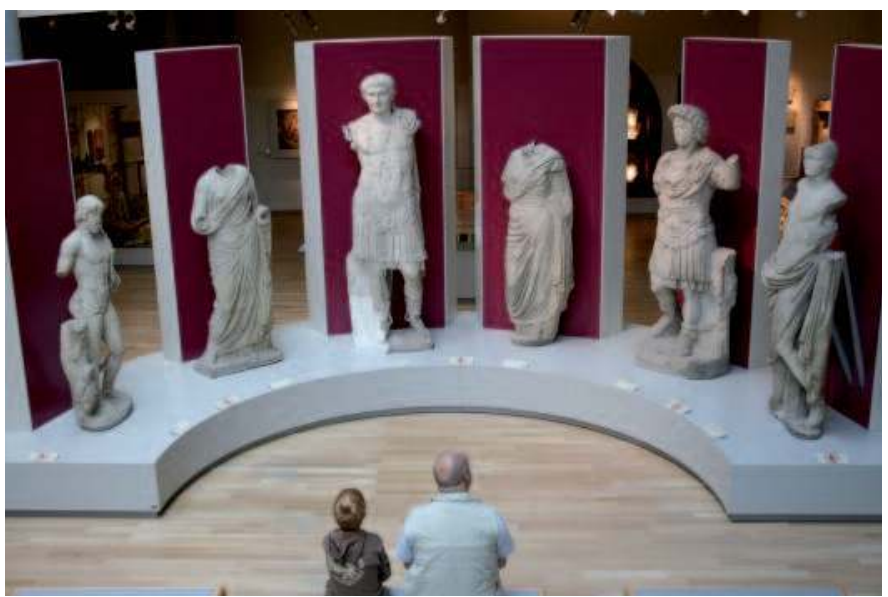

Les «Pèlerins respectueux » (c) Flickr/Vijaykiran

curieuse. D'autres visiteurs se servent du musée pour satisfaire des rôles ou des valeurs personnels comme celle d'être un bon parent ou un touriste culturel intrépide. Malgré les points communs entre ces self-aspects, les visiteurs individuels les utilisent comme l'expression de leur propre et unique identité. Cependant, la façon dont on se voit comme visiteur de musée dépend dans une large mesure de la façon dont on conceptualise le musée. Autrement dit, si vous vous considérez comme un bon père et que vous croyez que les musées sont le genre d'endroit où les bons pères amènent leurs enfants, alors vous pourriez rechercher activement un tel endroit afin de «promulguer » une telle identité. Ou, si vous pensez que vous êtes le genre de personne curieuse qui fait l'effort de découvrir des faits inhabituels et intéressants sur la condition humaine, à la fois dans le présent et le passé, alors vous pourriez rechercher activement un musée d'histoire pendant votre temps libre. En fait, un grand pourcentage de visiteurs de musées se comporte ainsi, non seulement en tant que parent ou curieux, mais aussi comme un moyen de se conformer à une identité.

Comme les musées sont devenus de plus en plus des lieux de loisir, beaucoup de personnes ont cherché à comprendre en quoi consistent les musées et comment elles peuvent les utiliser ; autrement dit, qu'est-ce que l'expérience de visite leur offre? Ces «offres» sont ensuite jumelées avec les besoins et les désirs liés à leur identité. Ensemble, elles créent une boucle de rétroaction forte et positive. La boucle commence par le public qui recherche des activités de loisir répondant à des besoins spécifiques liés à l'identité, tels que l'épanouissement personnel, la compétence de parent, ou la recherche de nouveauté. Comme les musées sont généralement perçus comme des endroits capables de répondre à certains besoins (mais pas tous) liés à l'identité, il devient pertinent de s'y rendre. Rétrospectivement, les visiteurs réfléchissent à leur visite de musée et jugent si l'expérience était un bon moyen de satisfaire leurs besoins ; et, si elle l'était, ils parlent à d'autres personnes de la visite, ce qui contribue à nourrir une représentation sociale estimant que ce musée et d'autres musées servent bien à cet effet. Par conséquent, ces visiteurs passés et d'autres comme eux sont beaucoup plus susceptibles de rechercher ce musée ou un autre à l'avenir s'ils possèdent un besoin semblable lié à cette identité.

\section{Une typologie de l'expérience de visite selon l'identité et les self-aspects}

De nombreuses études, dans différents musées, tendent à prouver l'existence de ces boucles de rétroaction liées à l'identité. Les façons dont les individus décrivent leurs expériences de visite semblent refléter les self-aspects liés à l'identité. Bien que potentiellement, il soit possible que les visiteurs de musée possèdent un nombre infini de self-aspects, cela ne semble pas être le cas. Les raisons que les gens donnent pour expliquer leurs motifs de visite et les descriptions après-musée de leur expérience ont eu tendance à se regrouper autour de seulement quelques catégories de base, qui à leur tour semblent refléter la façon dont le public perçoit ce qu'offre une visite.

En me fondant sur ces résultats, j'ai proposé le regroupement de tous les différents motifs que les visiteurs attribuent à la visite de musée en distinguant seulement cinq catégories différentes, liées à l'identité et aux self-aspects :

- les Explorateurs (Explorers) (5) : ce sont les visiteurs qui se piquent de curiosité avec un intérêt prépondérant pour le contenu du musée. Ils s'attendent à trouver quelque chose qui va capter leur attention et augmenter leurs connaissances.

"Je me souviens avoir pensé que je voulais ré-apprendre mes bases scientifiques, comme la biologie et tout ça... J'ai pensé [avant de venir], je ne vais pas tout saisir, vous savez, mais je vais apprendre certaines choses ».

- les Facilitateurs (Facilitators) : leur motivation relève de la sociabilité. Leur visite est principalement axée sur l'expérience et l'apprentissage des autres dans le groupe social qu'ils accompagnent.

" [Je suis venu] donner à [mes] enfants l'occasion de découvrir les débuts de la vie. C'est une bonne façon de passer du temps avec la famille d'une manière non commerciale. Ils apprennent toujours tant».

- les Professionnels/et Amateurs avertis (Professional/Hobbyists) : ils perçoivent un lien étroit entre le 
contenu du musée et leurs passions professionnelles ou leurs passe-temps favoris. Leurs visites sont généralement motivées par le désir de satisfaire un objectif lié au contenu.

"Je commence à mettre sur pied un aquarium récifal d'eau salée, donc je m'intéresse beaucoup à la vie marine. J'espère ramasser quelques idées [ici à l'aquarium]». - les Curieux d'expérience (Experience Seekers) : ils sont venus parce qu'ils perçoivent le musée comme une destination importante. Leur satisfaction provient essentiellement du simple fait d'avoir «été là ». "Nous venions d'ailleurs, et cherchions quelque chose d'amusant à faire qui ne prendrait pas toute la journée. Cela semblait être une bonne idée, après tout, nous sommes à Los Angeles et quelqu'un nous a dit que cet endroit vient d'ouvrir et qu'il est vraiment sympa».

- les Ressourceurs (Rechargers) : les visiteurs qui cherchent principalement à avoir une expérience contemplative, spirituelle et réparatrice. Ils voient le musée comme un refuge contre le monde métro-boulotdodo ou comme une confirmation de leurs croyances religieuses.

"J'aime les musées d'art. Ils sont si silencieux et relaxants, tellement différents $d u$ bruit et des encombrements $d u$ reste de la ville».

Au cours de l'année dernière, après avoir enquêté dans un plus large éventail d'établissements et de contextes culturels, dans des lieux particuliers tels que les musées anthropologiques ou axés sur les commémorations, j'ai proposé deux catégories supplémentaires (Bond et Falk, à paraître) :

- les Pèlerins respectueux (Respectful Pilgrims) : ils se rendent dans les musées par souci de devoir ou l'obligation d'honorer la mémoire de ceux qui sont célébrés par un établissement ou un monument.

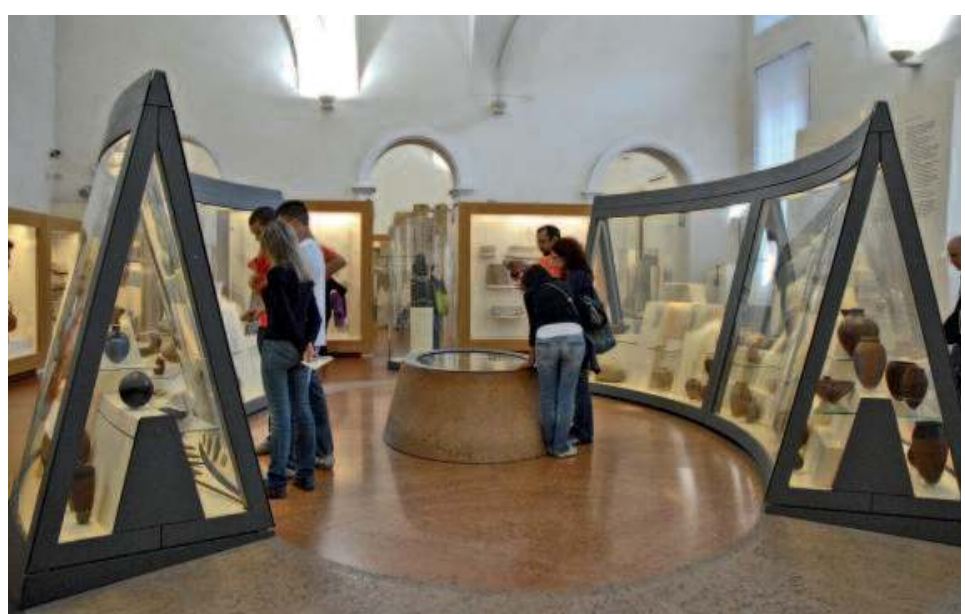

Les «Chercheurs d'affinité », au musée égyptien de Turin ( ) Flickr/Dalbera
- les Chercheurs d'affinité (Affinity Seekers) : ils sont attirés par un musée ou plus probablement une exposition qui leur fait penser à leur patrimoine ou leur rappelle leur personnalité.

Comme prévu, les visiteurs de musée se servent des musées pour satisfaire des besoins liés à l'identité, des identités profondément ancrées parfois, telles que la personne qui se voit avant tout comme une «personne qui aime l'art ». Mais aussi des identités plus communes ou même plus éphémères, comme la personne à la recherche d'une manière appropriée, pour elle, de passer un après-midi dans la ville qu'elle visite.

Ce qui est peut-être plus important, cependant, c'est que cette recherche a produit des preuves solides sur le fait que la catégorisation des visiteurs en fonction de leur perception des motifs de visite reliés à leur identité peut être utilisée comme un outil conceptuel. Il permet de relier des informations importantes sur la manière dont les visiteurs saisissent la signification de leur expérience de musée, à la fois avant d'arriver, au cours de l'expérience et ultérieurement quand ils repensent à leur visite. Dans notre dernière recherche, la majorité des visiteurs ne pouvait pas seulement être catégorisée comme appartenant à l'une de nos cinq catégories : les visiteurs au sein d'une catégorie se comportaient et apprenaient d'une façon différente de ceux appartenant à d'autres catégories (Falk, 2009). Plus précisément, les visiteurs de certaines catégories ont révélé des changements significatifs de leur compréhension et de leur sentiment, tandis que les visiteurs d'autres catégories ne les manifestent pas ; pour certaines catégories de visiteurs, l'expérience de visite est plutôt réussie, tandis que pour d'autres, elle ne l'est que de façon marginale.

Ainsi, contrairement aux stratégies de segmentation traditionnelle fondées sur les catégories socio-démographiques comme l'âge, l'appartenance à un groupe, le sexe, ou même le niveau de diplôme, en séparant les visiteurs en fonction de leurs motivations initiales liées à une identité, on dispose d'informations qui sont prédictives de l'expérience de visite. En outre, contrairement aux catégories socio-démographiques, ces catégories ne sont pas des qualités permanentes de l'individu. Un individu peut avoir envie d'aller au musée aujourd'hui parce qu'il veut promouvoir l'apprentissage de ses enfants et aller au même ou dans un autre demain car il sera en résonnance avec ses propres intérêts et sa curiosité personnelle. Selon les différents besoins dépendant d'une identité, la nature et la qualité de l'expérience de visite d'un même individu seront très différentes d'un jour à l'autre. 

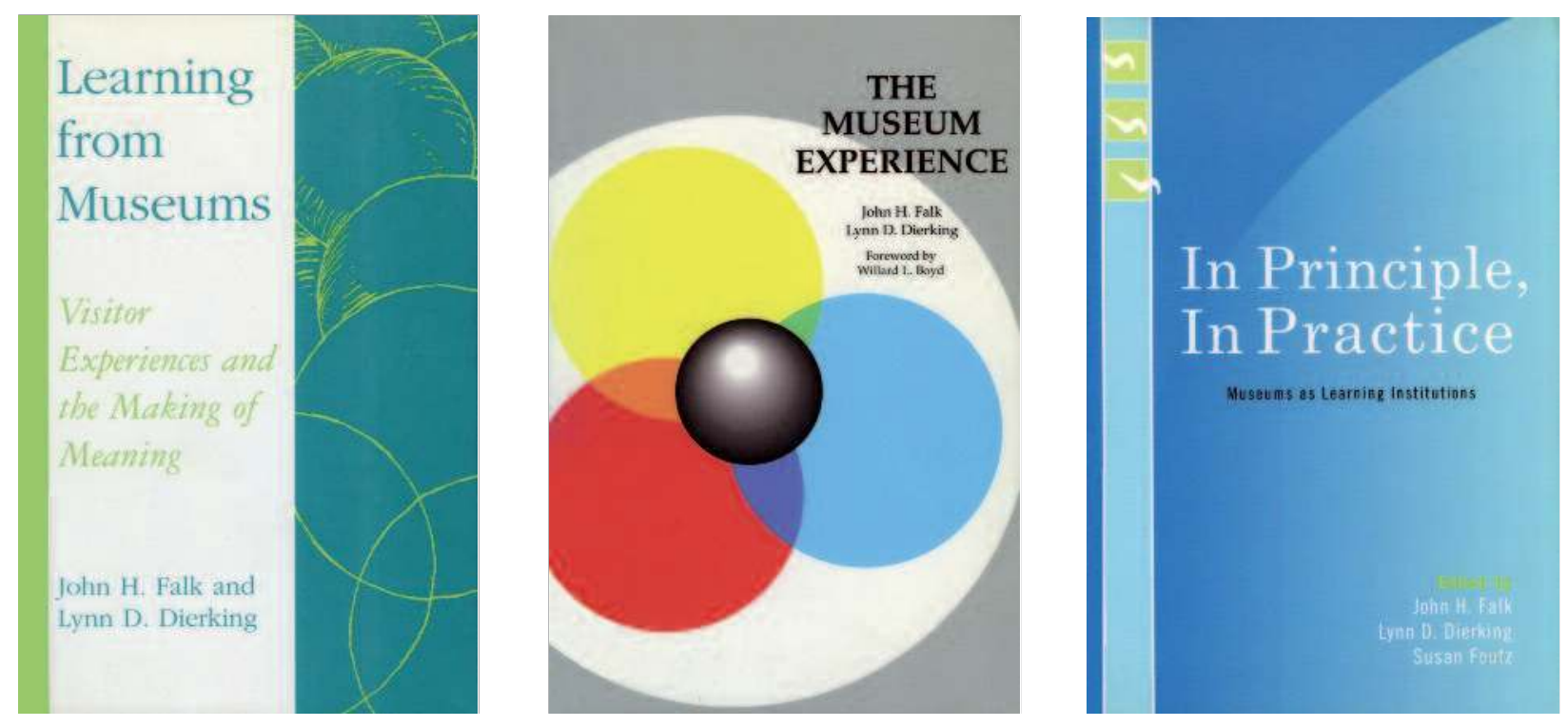

\section{Expérience de visite et recomposition de l'offre}

Quelles perspectives ouvrent ces hypothèses de recherche ? Non seulement, cette recherche révèle que la majorité des visiteurs arrive dans la plupart des types de musées avec des motivations différentes, mais de plus, il apparaît que les self-aspects liés à l'identité sont dépendants du musée, de son contenu, de comment les visiteurs se comportent et interagissent dans ce contexte et surtout, quelle signification ils retirent et mémorisent de l'expérience de visite une fois qu'ils ont quitté le lieu. En d'autres mots, être capable de segmenter les visiteurs de cette façon donne aux praticiens du musée des renseignements clés sur les besoins et les intérêts de leurs visiteurs. Ceci est très différent de la perspective «tailleunique » qui a historiquement orienté les interactions proposées aux visiteurs de musée.

Par exemple, ces recherches montrent que les « Explorateurs » sont concentrés sur ce qu'ils voient et trouvent intéressant, et mettent en œuvre ce programme centré sur soi indépendamment du fait qu'ils font partie d'un groupe social comme une famille avec des enfants ou non. Les «Facilitateurs " se concentrent sur ce que leurs proches voient et trouvent intéressant, et ils agissent en fonction de ce programme, par exemple, en permettant à un proche de diriger la visite et en s'inquiétant surtout de savoir si l'autre voit ce qu'il trouve intéressant, plutôt que de se préoccuper de ses propres intérêts. Les « Curieux d'expérience » sont enclins à réfléchir sur la forme de la journée, en particulier pour que la visite soit agréable. Les « Professionnels/Amateurs avertis » ont tendance à venir avec des intérêts très spécifiques, axés sur le contenu et à utiliser le musée comme un véhicule pour satisfaire ces intérêts (par exemple, l'information qui soutiendra leur propre collection personnelle ou la prise de photographies). Enfin, les « Ressourceurs", comme les «Curieux d'expérience », sont plus axés sur la forme de la journée. Mais à l'inverse des « Curieux d'expérience », les « Ressourceurs » n'ont pas tellement envie de s'amuser puisqu'ils préfèrent une expérience paisible ou inspirante. En se concentrant sur ces besoins et intérêts, les professionnels de musée pourraient personnaliser l'expérience de visite et donc satisfaire plus de visiteurs plus souvent.

Une autre conclusion importante de cette recherche est qu'elle prouve que l'expérience de visite " tailleunique » offerte par la plupart des musées (par exemple, des expositions, des programmes, des visites) ne fonctionne pas aussi bien tout le temps pour tous les visiteurs. Le contenu peut être pertinent pour certains, et totalement rater la cible pour les autres. En apprenant davantage sur les besoins spécifiques de chaque visiteur, ou au moins par catégories différentes, il devrait être possible de mieux répondre aux besoins de plus de visiteurs, plus souvent. Plus la relation entre la perception d'un visiteur de son expérience de visite proprement dite et sa perception identitaire de ses besoins augmente, plus il est probable que les visiteurs ressentent la qualité de leur visite et plus ils seront à même de revenir eux-mêmes au musée ou d'encourager les autres à le faire. 


\section{Notes des traducteurs}

(1) Nous avons délibérement sauté la variable « race » qui est couramment utilisée dans les enquêtes de publics aux États-Unis mais politiquement peu correcte en France et dans d'autres pays.

(2) Il va se soi que l'auteur ici ne conteste pas réellement la valeur explicative des variables sociologiques pour prédire, en moyenne constatée, la composition sociale des publics des musées et de la culture. Mais il essaie de compléter ces faits connus et d'aller au-delà pour décrire et formaliser la nature singulière de l'expérience de visite.

(3) Dans cet article, le mot «identité » est le concept central du modèle proposé par J.-H. Falk. Le concept d'identité désigne en pscychologie sociale « le point d'articulation entre la personnalité de l'individu, l'idée ou la représentation quill a de lui-même, et de l'ensemble des facteurs qui dans le contexte social dans lequel il s'inscrit, agissent sur lui ».

(4) Le self (en français le soi) est le mot anglais correspondant à la notion d'identité. Le mot anglais self-aspects désigne littéralement les aspects (ou les facettes ? les éléments ?) qui constituent le self (le soi). En réalité, les psycologues sociaux francophones ne traduisent ni self, ni self-aspects ce qui nous a conduit, dans ce passage en tout cas, à conserver les mots en anglais. (5) Il n'est jamais aisé de traduire les mots adoptés par un chercheur étranger pour désigner les catégories de visiteurs d'une typologie dite compréhensive. Généralement, on adopte des appellations plus ou moins métamorphiques et propres à une langue maternelle singulière. Nous avons donc traduit les noms des catégories de visiteurs en maintenant entre parenthèses les mots américains.

\section{Bibliographie}

American Association of Museums Data report : from the 1996 national museum survey. Washington, DC : American Association of Museums, 1998.

Bakhtin, M. The dialogic imagination : four essays. (edited by M. Holquist, translated by C. Emerson et M. Holquist). Austin : University of Texas Press, 1981.

Bourdieu, P. et Darbel, A. The love of art : european art museums and their public (C. Beattie et N. Merriman, Trans.). Cambridge : Polity Press, 1991/1969.

Bruner, J. et Kalmar, D.-A. Narrative and metanarrative in the construction of self, in M. Ferrari et R.-J. Sternberg (Eds.) Self-Awareness : its nature and development (pp. 308-331). New York : The Guildford Press, 1998.

Doering, Z.-D. et Pekarik, A. Questioning the entrance narrative, Journal of Museum Education 21(3) : 20-25, 1996.

Erikson, E.-H. Identity : Youth and crisis. New York : Norton, 1968.

Falk, J.-H. Factors Influencing Leisure Decisions : the Use of Museums by African Americans. Washington, DC : American Association of Museums, 1993.
Falk, J.-H. Identity and the Museum Visitor Experience. Walnut Creek, CA : Left Coast Press, 2009

Falk, J.-H., Koran, J.-J., Dierking, L.-D. et Dreblow, L. Predicting visitor behavior, Curator, 28 (4), 1985, pp. 326-332.

Falk, J.-H. et Needham, M. Measuring the impact of a science center on its community, Journal of Research in Science Teaching, 48 (1), 2011, pp. 1-12.

Falk, J.-H. et Storksdieck, M. Using the Contextual Model of Learning to understand visitor learning from a science center exhibition, Science Education, 89, 2005, pp. 744-778.

McGaugh, J.-L. Memory \& emotion : the making of lasting memories. New York : Columbia University Press, 2003.

Simon, B. Identity in modern society : a social psychological perspective. Oxford, UK : Blackwell, 2004 